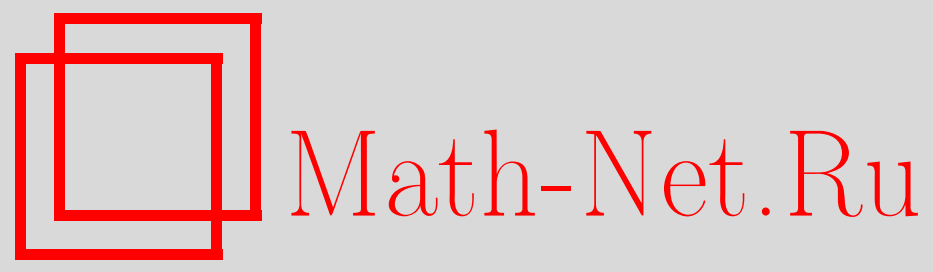

А. В. Чашкин, О нижних оценках сложности систем векторов $k$-значной логики, Дискрет. матем., 1998, том 10, выпуск 1, 46-62

DOI: https://doi.org/10.4213/dm407

Использование Общероссийского математического портала Math-Net.Ru подразумевает, что вы прочитали и согласны с пользовательским соглашением http: //www . mathnet.ru/rus/agreement

Параметры загрузки:

IP: 54.162 .85 .209

26 апреля 2023 г., 16:39:23 


\title{
О нижних оценках сложности систем векторов $k$-значной логики
}

\author{
(c) 1998 г. А. В. Чашкин
}

\begin{abstract}
Исследуется сложность порождения систем векторов схемами в базисах $B_{k, 1}, B_{k, 2}, B_{k, 3}$, где $B_{k, 1}$ - множество всех не более чем двуместных операций $k$-значной логики, $B_{k, 2}-$ множество всех не более чем одноместных операций $k$-значной логики, содержащее также двуместные операции $\max$ и $\min , B_{k, 3}$ - множество всех не более чем одноместных монотонных операций $k$-значнон логики, содержащее также двуместные операции $\max$ и $\min$.

Основными результатами работы являются нижние оценки сложности порождения систем векторов и соотношения, связывающие нижние оценки сложности порождения систем вехторов и нижние оценхи сложности функций $k$ значной логики. Из этих соотношений следует, что установление нижних оценок сложности порождения узких систем векторов, асимптотически больших, чем полученные в этой работе, влечет установление экспоненциальных нижних оценок сложности функции $k$-значноң логики при их реализации схемами в полных базисах. Под узкими системами понимаются системы, у которых число векторов по порядку не превосходит логарифма числа компонент этих векторов. Ранее автором были получены аналогичные результаты для систем булевых векторов.
\end{abstract}

Работа выполнена при поддержке Россииского фонда фундаментальных исследовании, грант 96-01-01068.

\section{1. Введение}

Множество $n$-мерных векторов $k$-значной логики $\{0,1, \ldots, k-1\}^{n}$ обозначим через $E_{k}^{n}$. Пусть $G \subseteq E_{k}^{n},|G|=m, B \subseteq\left\{f: E_{k}^{n} \rightarrow E_{k}^{1}\right\}$.

$(B, G)$-схемой называется ориентированный ациклический граф $S$, обладающий следующими свойствами.

Граф $S$ содержит $m$ вершин с входной степенью, равной нулю, и каждой из этих вершин приписан некоторый вектор $g$ из $G$, причем разным вершинам приписаны разные векторы. Такие вершины называются входами схемы. Множество $G$ называется множеством генераторов схемы.

Входные степени остальных вершин не превосходят двух. Эти вершины называются элементами схемы. Каждому элементу схемы с входной степенью, равной $j$, $j=0,1,2$, приписана $j$-местная операция из $B$. Множество $B$ называется базисом схемы. 
Если вершины $w$ и $u$ схемы $S$ связаны ребром, ориентированым от $u$ к $w$, то будем говорить что выход $u$ подключен к $w$ (или к входу $w$ ).

Сложностью схемы $S$ назовем число $|S|$ элементов этой схемы.

Для каждой вершины $w$ схемы $S$ определим вектор $S(w)$, порождаемый в этой вершине. Сделаем это индуктивно.

(1) Если $w-$ вход схемы, которому приписан вектор $g$, то $S(w)=g$.

(2) Если вершине $w$ приписана нульместная операция $f$, то $S(w)$ - постоянный вектор, все компоненты которого равны значению $f$.

(3) Если вершине $w$ приписана одноместная операция $f$ и к $w$ подключен выход вершины $u$, то $S(w)=f(S(u))$.

(4) Если вершине $w$ приписана двуместная операция $f$ и к $w$ подключены выходы вершин $u$ и $v$, то $S(w)=f(S(u), S(v))$.

Будем говорить что схема $S$ порождает систему векторов $W=\left\{w_{i}\right\}_{i=1}^{p}$, если в схеме $S$ найдется $p$ вершин $u_{i}$ таких, что $S\left(u_{i}\right)=w_{i}, i=1, \ldots, p$. Еслv среди всех схем, порождающих систему $W$, схема $S$ содержит наименьшее число элементов, то $S$ называется минимальной схемой.

Число $V_{B, G}(W)$ назовем $(B, G)$-сложностью системы векторов $W$, если оно равно сложности минимальной схемы, порождающей $W$. Если множество генераторов $G$ состоит из всех векторов, единственная ненулевая компонента которых равна единице, $G=\left\{e_{i}\right\}_{i=1}^{n}$, то, говоря о $(B, G)$-сложности системы векторов $W$, будем опускать символ $G$ и обозначать эту сложность через $V_{B}(W)$.

В работе исследуется сложность порождения систем векторов схемами в базиcax $B_{k, 1}, B_{k, 2}, B_{k, 3}$, где $B_{k, 1}$ - множество всех не более чем двуместных операций $k$-значной логики, $B_{k, 2}$ - множество всех не более чем одноместных операций $k$ значной логики, содержащее также двуместные операции $\max$ и $\min , B_{k, 3}$ - множествс всех не более чем одноместных монотонных операций $k$-значной логики, содержащее также двуместные операции $\max$ и $\min$. Операцию $\max$ дем иногда обозначать через $V$.

Матрицей системы векторов

$$
\left\{w_{i}=\sum_{j} w_{i j} e_{j}\right\}_{i=1}^{p}
$$

назовем матрицу $W$ с элементами $W_{i, j}=w_{i j}$ при всех $i$ и $j$. Далее при доказательстве различных утверждений вместо систем векторов будем использовать матрицы этих систем. Такая замена часто позволяет упростить изложение.

Основными результатами работы являются доказанные в леммах 2-4 нижние оценки сложности порождения систем векторов и доказанные в теоремах 4-6 соотношения, связывающие нижние оценки сложности порождения систем векторов и нижние оценки сложности функций $k$-значной логики. Из этих соотношений следует, что установление нижних оценок сложности порождения узких систем векторов, асимптотически больших, чем полученные в леммах 2-4, влечет установление экспоненциальных нижних оценок сложности функций $k$-значной логики при их реализации схемами в полных базисах. Здесь под узкими системами понимаются 
системы, у которых число векторов по порядку не превосходит логарифма числа компонент этих векторов. Ранее аналогичные результаты для систем булевых векторов были получены автором в [1]. Неопределяемые в работе понятия можно найти B [2].

\section{2. Нижние оценки сложности}

Лемма 1. Пусть схема $S$ содержит

(1) р вериин со входной степенъю, равной нулю,

(2) $q$ вершин со входной степенъю, равной единиче,

(3) не более $r$ вершин с въходной степенъю, равной нулю,

(4) не менее $s$ вершин с выходной степенъю, равной двум.

Тогда схема $S$ содержит не менее $p+q+s-r$ элементов.

Доказательство. Сравним количество входящих и выходящих ребер схемы $S$. Число вершин схемы $S$ обозначим через $t$. Легко видеть, что $S$ содержит $2(t-p-q)+q$ входящих ребер и не менее $(t-r-s)+2 s$ выходящих ребер. Следовательно,

$$
2(t-p-q)+q \geqslant(t-r-s)+2 s \text {. }
$$

Тогда

$$
|S| \geqslant(t-p) \geqslant p+q+s-r .
$$

Лемма доказана.

Вершину $и$ схемы $S$ назовем особой вершиной схемы $S$, если либо выходная степень $u$ больше единицы, либо если $u$ - выход $S$.

Особую вершину $u$ схемы $S$ назовем особой вершиной $i$-го входа $S$, если $i$-й вход и $u$ связаны ориентированной цепью, не проходящей через другие особые вершины.

Если вершины $w$ и $u$ схемы $S$ связаны цепью, ориентированной от $u$ к $w$, то будем говорить что вершина $w$ находится ниже вершины $u$.

Схему $S$ в базисе $B_{k, 1}$ назовем нормальной, если $S$ содержит только двухвходовые элементы. Схему $S$ в базисе $B_{k, 2}$ назовем нормальной, если $S$ содержит только одновходовые и двухвходовые элементы. Легко показать, что любая схема как в базисе $B_{k, 1}$, так и в базисе $B_{k, 2}$ может быть преобразована в нормальную схему без увеличения сложности.

Будем полагать что к $i$-му входу всех рассматриваемых ниже $(B)$ - схем приписан вектор $e_{i}$.

Преобразование схемы $S$ в базисе $B_{k, 1}\left(B_{k, 2}\right)$, заключающееся в замене вектора, подключеного к $i$-му входу, нулевым вектором, удалении $i$-й компоненты из всех векторов, подключеных к входам схемы, и пребразование $S$ в нормальную схему назовем удаленем $i$-го входа. Легко показать, что сложность любой схемы, полученой в результате удаление произвольного неизолированого входа, не больше сложности исходной схемы.

Пусть $W(k, n, m)$-- множество всех матриц $k$-значной логики размера $n \times m$, состоящих из попарно различных столбцов. 
Лемма 2. Пусть $W \in W(k, n, m)$. Тогда

$$
\left.V_{B_{k, 1}}(W) \geqslant m-1+\right](m-1) /(k-1)[-2 n .
$$

Доказательство. Пусть $W$ - множество матриц, имеющих минимальную сложность среди всех матриц множества $W(k, n, m)$. Покажем, что среди минимальных схем, порождающих матрицы этого множества, обязательно найдется схема с изолированным входом. Предположим, что это не так. Пусть $W_{0}$ - произвольная матрица из $W$. Преобразуем некоторую минимальную схему $S_{0}$, порождающую эту матрицу, следующим образом:

- добавим к схеме новый $(m+1)$-й изолированный вход,

- увеличим длину всех генераторов на единицу, добавив к каждому из них $(m+1)$-ю нулевую компоненту.

Преобразованная схема $S_{0}^{\prime}$ будет порождать некоторую матрицу $k$-значной логики $W_{0}^{\prime}$ размера $n \times(m+1)$. Возможны два случая:

$W_{0}^{\prime}$ принадлежит множеству $W(k, n, m+1)$,

в $W_{0}^{\prime}$ найдутся два одинаковых столбца, $(m+1)$-й и некоторый $j$-й.

Если имеет место первый случай, то удалим из схемы $S_{0}^{\prime}$ произвольный неизолированный вход. Новая схема $S_{0}^{\prime \prime}$ будет порождать некоторую матрицу $W_{0}^{\prime \prime}$. Очевидно, что $W^{\prime \prime}$ принадлежит множеству $W(k, n, m)$ и

$$
V_{B_{k, 1}}\left(W_{0}^{\prime \prime}\right) \leqslant V_{B_{k, 1}}\left(W_{0}^{\prime}\right)
$$

Последнее неравенство противоречит сделанному предположению, и, следовательно, первый случай невозможен.

Во втором случае из схемы $S_{0}^{\prime}$ удалим $j$-й вход. Новая схема $S_{0}^{\prime \prime \prime}$ будет порождать матрицу $W_{0}^{\prime \prime \prime}$, отличающуюся от матрицы $W_{0}$ только порядком следования столбцов. Очевидно, что $\left|S_{0}^{\prime \prime \prime}\right| \leqslant\left|S_{0}\right|$, а это вновь противоречит сделанному предположению. Следовательно, второй случай также невозможен, и поэтому обязательно найдется схема, содержащая изолированный вход. Без ограничения общности будем полагать, что таким входом является первый вход.

Теперь покажем что в схеме $S_{0}$ никакие $k$ входов не могут иметь общую особую вершину. Предположим, что это не так, и некоторая вершина $u$ является особой вершиной входов $i_{1}, i_{2}, \ldots, i_{k}$ (заметим, что ни один из этих входов не равен единице, так как изолированным по предположению является перзый вход). Очевидно, что среди компонент вектора $S_{0}(u)$, соответствующих первому входу и входам $i_{j}$, $j=1, \ldots, k$, найдется не менее двух одинаковых компонент. Но тогда эти компоненты будут одинаковы и во всех других векторах, порождаемых элементами схемы $S_{0}$, находящимися ниже $u$. Это следует из того, что все ориентированные цепи, соединяющие входы $i_{j}$ с этими элементами, проходят через вершину $u$. Следовательно, матрица, порождаемая схемой $S_{0}$, будет содержать не менее двух одинаковых столбцов, а это противоречит определению матрицы $W_{0}$.

Из выявленных выше свойств схемы $S_{0}$ следует, что в этой схеме найдется не менее $](m-1) /(k-1)$ [ особых вершин, и по крайней мере ] $(m-1) /(k-1)[-n$ из них не являются выходами $S_{0}$. Следовательно, в схеме $S_{0}$ содержится не менее 
]$(m-1) /(k-1)[-n$ вершин, выходная степень которых не менее двух. В соответствии с леммой 1

$$
\left.\left|S_{0}\right| \geqslant m-(n+1)+(](m-1) /(k-1)[-n)=m-1+\right](m-1) /(k-1)[-2 n .
$$

Лемма доказана.

Лемма 3. Пусть $W \in W(k, n, m)$. Тогда

$$
V_{B_{k, 2}}(W) \geqslant 2 m-2 n-2 .
$$

Доказательство. Пусть $W$ - множество матриц, имеющих минимальную сложность среди всех матриц множества $W(k, n, m)$. Как и при доказательстве предыдущей леммы, несложно показать, что в $W$ обязательно найдется матрица, одна из минимальных схем которой содержит по крайне мере один изолированный вход. Эту схему обозначим через $S_{0}$. Как и ранее, полагаем, что изолированным является первый вход.

Теперь покажем, что в схеме $S_{0}$ никакие два входа не могут иметь общую особую вершину. Предположим, что это не так и некоторая вершина $u$ является особой вершиной входов $i_{1}$ и $i_{2}$. Очевидно, что среди компонент вектора $S_{0}(u)$, соответствующих первому входу и входам $i_{j}$, найдется не менее двух одинаковых компонент. Это связано с тем, что цепи, соединяющие каждый из входов $i_{j}$ с $u$, проходят только через элементы, которым приписаны $\max$ или $\min$. Но тогда эти компоненты будут одинаковы и во всех других векторах, порождаемых элементами схемы $S_{0}$, находящимися ниже $u$. Следовательно, матрица, порождаемая схемой $S_{0}$, будет содержать не менее двух одинаковых столбцов, а это противоречит определению матрицы $W_{0}$.

Из выявленных выше свойств схемы $S_{0}$ следует, что в этой схеме найдется не менее $m-1$ особых вершин и по крайней мере $m-1-n$ из них не являются выходами $S_{0}$. Следовательно, в схеме $S_{0}$ есть не менее $m-1-n$ вершин, выходная степень которых не менее двух. В соответствии с леммой 1

$$
\left|S_{0}\right| \geqslant m-(n+1)+(m-1-n)=2 m-2 n-2 .
$$

Лемма доказана.

Пусть $W^{\prime}(k, n, m)$ - множество всех матриц $k$-значной логики размера $n \times m$, состоящих из попарно несравнимых столбцов.

Лемма 4. Пусть $W \in W^{\prime}(k, n, m)$. Тогда

$$
V_{B_{k, 3}}(W) \geqslant 2 m-2 n-2 .
$$

Доказательство. Пусть $S$ - минимальная схема, порождающая матрицу $W$. Покажем, что в схеме $S$ никакие два входа не могут иметь общую особую вершину. Предположим, что это не так и некоторая вершина $u$ является особой вершиной входов $i_{1}$ и $i_{2}$. Очевидно, что либо компоненты вектора $S(u)$, соответствующие входам $i_{j}$, будут одинаковы, либо одна из них будет меньше другой. В первом случае 
эти компненты будут одинаковы и во всех других векторах, порождаемых элементами схемы $S$, находящимися ниже $u$. Следовательно, матрица, порождаемая схемой $S$, будет содержать не менее двух одинаковых столбцов, а это противоречит определению матрицы $W$. Во втором случае компонента, меньшая в $S(u)$, будет не больше и во всех векторах, порождаемых элементами схемы $S$, находящимися ниже $u$. Это следует из монотонности базиса схемы. Следовательно, матрица, порождаемая схемой $S$, будет содержать не менее двух сравнимых столбцов, а это противоречит определению матрицы $W$.

Как и при доказательстве предыдущей леммы, заключаем, что в схеме $S$ найдется не менее $m-1$ особых вершин, не менее $m-1-n$ из которых не являются выходами $S$. Использование леммы 1 завершает доказательство.

\section{3. Верхние оценки сложности}

Обозначим через $f_{k}(\alpha, \beta)$ и $g_{k}(\alpha, \beta)$ произвольные функции $k$-значной логики, удовлетворяющие равенствам

$$
\begin{aligned}
& f_{k}(0, \alpha)= \begin{cases}\alpha+1, & \text { если } 1 \leqslant \alpha \leqslant k-2, \\
1, & \text { если } \alpha \leqslant k-1,\end{cases} \\
& f_{k}(\alpha, 0)=\alpha \\
& g_{k}(\alpha, 0)=g_{k}(0, \alpha)=\alpha .
\end{aligned}
$$

Схему $S$ назовем разделимой, если любой вход $S$ связан с любым выходом $S$ не более, чем одной ориентированной цепью.

Положим

$$
D_{k}=\left\{f_{k}, g_{k}\right\} \text {. }
$$

Лемма 5. Пустъ $S$ - разделимая схема в базисе $D_{k}, W$ - матрица, порождаемая схемой $S, t_{i j}$ - число вторых входов элементов $f_{k}$, через которые проходит, цепъ соединяющая $j$-й вход и $i$-й въход схемы $S$. Тогда

$$
W_{i, j}= \begin{cases}0, & \text { если нет чепи, соединяющей } j \text {-й вход } u \text { i-й вход }, \\ 1+\left(t_{i j} \bmod (k-1)\right) & \text { в противном случае. }\end{cases}
$$

Для доказательства леммы достаточно рассмотреть цепь, соединяющую $j$-й вход и $i$-й выход схемы (если такая цепь есть) и определить, как преобразуют элементы, через которые эта цепь проходит, $j$-ю компоненту вектора, подключенного к $j$-му входу.

Пусть $B(k, n)$ - матрица размера $(n+1) \times k^{n}$, первые $n$ строк которой образуют некоторую матрицу $W$ из множества $W\left(k, n, k^{n}\right)$, а последняя строка содержит только единицы. Без ограничения общности будем считать, что $j$-й столбец матрицы $W$ совпадает с $k$-ичным разложением числа $j-1$.

Лемма 6. Существует разделимая схема $S$ в базисе $D_{k}$, порождающая матричу $B(k, n)$, такая, что

$$
|S| \leqslant 2\left(k^{n}-1\right)-n
$$


Доказательство. Построим разделимую схему $S_{k, n}$, порождающую матрицу $B(k, n)$ и имеющую сложность $2\left(k^{n}-1\right)-n$. Строить схему $S_{k, n}$ при произвольных $k \geqslant 2$ и $n \geqslant 1$ будем индукцией по $n$. Основанием индукции будет схема $S_{k, 1}$. Эта схема порождает первую строку матрицы $B(k, 1)$ в соответствии с формулой

$$
f_{k}\left(x_{2}, f_{k}\left(x_{3}, \ldots, x_{k-2}, f\left(x_{k-1}, x_{k}\right) \ldots\right),\right.
$$

вторую строку в соответствии с формулой

$$
g_{k}\left(x_{1}, g_{k}\left(x_{3}, \ldots, x_{k-2}, g\left(x_{k-1}, x_{k}\right) \ldots\right) .\right.
$$

Очевидно, что

$$
\left|S_{k, 1}\right|=2 k-3=2(k-1)-1,
$$

то есть сложность схемы $S_{k, 1}$ удовлетворяет неравенству леммы.

Предположим далее, что при некотором $n \geqslant 1$ и при произвольном $k \geqslant 2$ существует схема $S_{k, n}$, порождающая матрицу $B(k, n)$ с требуемой сложностью. На основе этой схемы построим схему $S_{k, n+1}$, порождающую матрицу $B(k, n+1)$. Cxема $S=S_{k, n+1}$ будет состоять из подсхем $S_{1}, S_{2}, S_{3}, S_{4}$. Опишем эти подсхемы.

Подсхема $S_{1}$ порождает все векторы

$$
y_{i}=\bigvee_{j} x_{j}, \quad(i-1) k^{n}<j \leqslant i k^{n}, \quad 2 \leqslant i \leqslant k .
$$

Подсхема $S_{2}$ порождает все векторы

$$
z_{i}=\bigvee_{j} x_{i+j k^{n}}, \quad 0 \leqslant j \leqslant k-1, \quad 1 \leqslant i \leqslant k^{n},
$$

Подсхема $S_{3}$ является экземпляром схемы $S_{k, n} . \mathrm{K} i$-му входу $S_{3}$ подключается вектор $z_{i}$, порождаемый подсхемой $S_{2}$. Легко видеть, что подсхема $S_{3}$ порождает последние $n$ строк матрицы $B(k, n+1)$.

Подсхема $S_{4}$ порождает первую строку матрицы $B(k, n+1)$, исходя из векторов $y_{i}$, порожденных подсхемой $S_{1}$, в соответствии с формулой

$$
f_{k}\left(y_{2}, f_{k}\left(y_{3}, \ldots, y_{k-2}, f\left(y_{k-1}, y_{k}\right) \ldots\right) .\right.
$$

Оценим сложность схемы $S$. Легко видеть, что

$$
\begin{aligned}
|S|=\sum_{i=1}^{4}\left|S_{i}\right| & =\left(k^{n}-1\right)(k-1)+k^{n}(k-1)+\left|S_{k, n}\right|+(k-2) \\
& =\left|S_{k, n}\right|+2\left(k^{n+1}-k^{n}\right)-1 .
\end{aligned}
$$

По предположению индукции

$$
\left|S_{k, n}\right|=2\left(k^{n}-1\right)-n,
$$

поэтому

$$
\left|S_{k, n+1}\right|=2\left(k^{n}-1\right)-n+2 k^{n}(k-1)-1=2\left(k^{n+1}-1\right)-(n+1) .
$$

Очевидно, что $S_{k, n+1}$ - разделимая схема.

Лемма доказана. 
Пусть $k^{n-1}<m<k^{n}, C(n, m)$ - матрица размера $(n+1) \times m$, образованная первыми $m$ столбцами матрицы $B(k, n)$.

Лемма 7. Существует разделимая схема $S$ в базисе $D_{k}$, порождающая матрииу $C(n, m)$, такая, чmo

$$
|S| \leqslant 2(m-1)-n \text {. }
$$

Доказательство. Положим $n^{\prime}=\left[\log _{k} m\right]$. Пусть $S^{\prime}-$ построенная в доказательстве леммы 6 схема, порождающая матрицу $B\left(k, n^{\prime}\right)$. Воспользуемся этой схемой для построения схемы $S$, порождающей матрицу $C(n, m)$. Схема $S$ состоит из подсхем $S_{1}, S_{2}, S_{3}, S_{4}$. Опишем эти подсхемы.

Подсхема $S_{1}$ состоит из элементов $g_{k}$ и порождает все векторы

$$
\left.y_{i}=\bigvee x_{j}, \quad(i-1) k^{n^{\prime}}<j \leqslant \min \left(i k^{n^{\prime}}, m\right), \quad 2 \leqslant i \leqslant l, \quad l=\right] m k^{-n}[.
$$

Подсхема $S_{2}$ состоит из элементов $g_{k}$ и порождает все векторы

$$
z_{i}=\bigvee_{j} x_{i+j k^{n^{\prime}}}, \quad 0 \leqslant j \leqslant k-1, \quad i+j k^{n^{\prime}} \leqslant m, \quad 1 \leqslant i \leqslant k^{n^{\prime}} .
$$

Подсхема $S_{3}$ является экземпляром схемы $S^{\prime}$. К $i$-му входу $S_{3}$ подключается вектор $z_{i}$, порождаемый подсхемой $S_{2}$. Легко видеть, что подсхема $S_{3}$ порождает последние $n^{\prime}+1$ строк матрицы $C(n, m)$.

Подсхема $S_{4}$ порождает первую строку матрицы $C(n, m)$, исходя из векторов $y_{i}$, порожденных подсхемой $S_{1}$, в соответствии с формулой

$$
f_{k}\left(y_{2}, f_{k}\left(y_{3}, \ldots, y_{k-2}, f\left(y_{k-1}, y_{k}\right) \ldots\right)\right. \text {. }
$$

Оценим сложность схемы $S$. Ясно, что

$$
\begin{aligned}
|S|= & \sum_{i=1}^{4}\left|S_{i}\right| \\
= & \left.m-k^{n^{\prime}}-\right]\left(m-k^{n^{\prime}}\right) k^{-n^{\prime}}\left[+(] m k^{-n^{\prime}}[-1)\left(m-(] m k^{-n^{\prime}}[-1) k^{-m^{\prime}}\right.\right. \\
& +(] m k^{-n^{\prime}}[-2)\left(k^{n^{\prime}}-\left(m-(] m k^{-n^{\prime}}[-1)\right)+2 k^{n^{\prime}}-n^{\prime}-2+\right]\left(m-k^{-n^{\prime}}\right) k^{-n^{\prime}}[-1 \\
= & 2 m-\left(n^{\prime}+1\right)-2=2(m-1)-n .
\end{aligned}
$$

Лемма доказана.

Пусть $T_{j}$ - множество индексов, определяемых посредством равенств

$$
T_{j}=\left\{i \mid\left(k^{j}-1\right) /(k-1)<i \leqslant\left(k^{j+1}-1\right) /(k-1)\right\}, \quad 0 \leqslant j \leqslant n .
$$

Обозначим через $B^{\prime}(k, n)$ матрицу размера $(n+1) \times\left(\left(k^{n+1}-1\right)(k-1)\right) k$-значной логики такую, что подматрица $B_{s}^{\prime}(k, n)$ этой матрицы, образованная первыми $s$ строками и столбцами, номера которых принадлежат множеству $T_{s-1}$, совпадает с матрицей $B(k, s-1)$, а подматрица, образованная этими же столбцами и последними $n+1-s$ строками состоит только из нулей. 
Лемма 8. Существует раздєлимая схема $S$ в базисе $D_{k}$, порождающая матрицу $B^{\prime}(k, n)$, такая, чтоо

$$
|S| \leqslant 2 \frac{k^{n+1}-1}{k-1}-2(n+1) .
$$

Доказательство. Построим схему $S_{k, n}^{\prime}$, порождающую матрицу $B^{\prime}(k, n)$ и имеющую сложность, равную $2\left(k^{n+1}-1\right) /(k-1)-2(n+1)$. Строить схему $S_{k, n}^{\prime}$ при произвольных $k \geqslant 2, n \geqslant 0$ будем индукцией по $n$ на основе схем $S_{k, n}$, приведенных в доказательстве леммы 6 . Основанием индукции будет схема $S_{k, 0}^{\prime}$, порождающая матрицу $B^{\prime}(k, 0)$. Легко видеть, что матрица $B^{\prime}(k, 0)$ состоит из единственной единичной компоненты и поэтому $V_{D_{k}}\left(B^{\prime}(k, 0)\right)=0$.

Предположим теперь, что при некотором $n \geqslant 0$ и произвольном $k \geqslant 2$ построена схема $S_{k, n}^{\prime}$, порождающая с требуемой сложностью матрицу $B^{\prime}(k, n)$. Воспользуемся этой схемой для построения схемы $S_{k, n+1}^{\prime}$. Схема $S_{k, n+1}^{\prime}$ состоит из подсхем $S_{1}$, $S_{2}, S_{3}$.

Подсхема $S_{1}$ является экземпляром схемы $S_{k, n}^{\prime}$, к $i$-му входу которой подключен вектор $x_{i}$.

Подсхема $S_{2}$ является экземпляром схемы $S_{k, n+1}$, к $i$-му входу которой подключен вектор $x_{i+\left(k^{n}-1\right) /(k-1)}$.

Подсхема $S_{3}$ состоит из элементов $s_{1}, s_{2}, \ldots, s_{n+1}$, каждый из которых выполняет операцию $g_{k}$. При этом к одному из входов каждого элемента $s_{i}$ подключен $i$-й выход подсхемы $S_{1}$, а к другому $i$-й выход подсхемы $S_{2}$.

Очевидно, что

$$
\left|S_{k, n+1}^{\prime}\right|=\sum_{i=1}^{3}\left|S_{i}\right|=\left|S_{k, n}^{\prime}\right|+\left|S_{k, n+1}\right|+(n+1) .
$$

Учитывая предположение индукции и неравенство леммы 6 видим, что

$$
\left|S_{k, n+1}^{\prime}\right|=2 \frac{k^{n+2}-1}{k-1}-2(n+2) .
$$

Лемма доказана.

Пусть

$$
\frac{k^{n}-1}{k-1}<m<\frac{k^{n+1}-1}{k-1}
$$

$C^{\prime}(n, m)$ - матрица размера $(n+1) \times m$, образованная первыми $m$ столбцами матрицы $B^{\prime}(k, n)$.

Лемма 9. Существует разделимая схема $S$ в базисе $D_{k}$, порождающая матричу $C^{\prime}(n, m)$, такая, что

$$
|S| \leqslant 2 m-2(n+1) \text {. }
$$

Доказательство. Пусть

$$
k^{t-1}<m-\frac{k^{n}-1}{k-1}<k^{t} .
$$

Пусть далее $S^{\prime}$ - построенная в доказательстве леммы 8 схема, порождающая матрицу $B^{\prime}(k, n-1)$, а $S^{\prime \prime}$ - построенная в доказательстве леммы 7 схема, порождающая матрицу $C\left(t, m-\left(k^{n}-1\right)(/(k-1))\right.$. Воспользуемся этими схемами для 
построения схемы $S$, порождающей матрицу $C^{\prime}(n, m)$. Схема $S$ состоит из подсхем $S_{1}, S_{2}, S_{3}$.

Подсхема $S_{1}$ является экземпляром схемы $S^{\prime}$, к $i$-му входу которой подключен вектор $x_{i}$.

Подсхема $S_{2}$ является экземпляром схемы $S^{\prime \prime}$, к $i$-му входу которой подключен вектор $x_{i+\left(k^{n}-1\right) /(k-1)}$.

Подсхема $S_{3}$ состоит из элементов $s_{1}, s_{2}, \ldots, s_{t}$, каждый из которых выполняет операцию $g_{k}$. При этом к одному из входов каждого элемента $s_{i}$ подключен $i$-й выход подсхемы $S_{1}$, а к другому $i$-й выход подсхемы $S_{2}$.

Очевидно, что

$$
\begin{aligned}
|S| & =\sum_{i=1}^{3}\left|S_{i}\right|=\left|S^{\prime}\right|+\left|S^{\prime \prime}\right|+t \\
& =2 \frac{k^{n}-1}{k-1}-2 n+2\left(m-\frac{k^{n}-1}{k-1}-1\right)-t+t \\
& =2 m-2(n+1) .
\end{aligned}
$$

Лемма доказана.

Теорема 1. При любом $m>1$ существует такая матрица $W \in W(k,] \log _{k} m[, m)$, чmo

$$
\left.V_{B_{k, 1}}(W)=m-1+\right](m-1) /(k-1)[-2] \log _{k} m[
$$

Доказательство. Для доказательства теоремы построим схему $S$ и покажем, что построенная схема порождает требуемую матрицу.

Пусть $m=(k-1) m_{1}+1+m_{2}$, где $0 \leqslant m_{2} \leqslant k-1$. Схема $S$ состоит из трех подсхем $S_{1}, S_{2}, S_{3}$.

Подсхема $S_{1}$ порождает $m_{1}+1$ различный вектор $h_{s}$ :

$$
h_{s}=f_{k}\left(x_{1+s(k-1)}, f_{k}\left(x_{2+s(k-1)}, \ldots, f_{k}\left(x_{k-2+s(k-1)}, x_{k-1+s(k-1)}\right) \ldots\right)\right)
$$

при $s=0,1, \ldots, m_{1}-1$ и при $s=m_{1}$

$$
h_{m}=f_{k}\left(x_{1+s(k-1)}, f_{k}\left(x_{2+s(k-1)}, \ldots, f_{k}\left(x_{m_{2}-2+s(k-1)}, x_{m_{-} 1+s(k-1)}\right) \ldots\right)\right) .
$$

Очевидно, что $\left|S_{1}\right|=m_{1}(k-2)+m_{2}-1$.

Подсхема $S_{2}$ является экземпляром построенной в доказательстве леммы 9 (или леммы 8 , если $m_{2}=k-1$ ) схемы $S$, порождающей матрицу $C^{\prime}\left(n, m_{1}+1\right)$ (или матрицу $\left.C\left(n, m_{1}+1\right)\right)$, к $s$-му входу которой подключен $s$-й выход подсхемы $S_{1}$. Так как $\left.m_{1}=\right](m-1) /(k-1)[-1$, то

$$
\begin{gathered}
\left.\frac{k^{n}-1}{k-1}<\right](m-1) /(k-1)\left[\leqslant \frac{k^{n+1}-1}{k-1},\right. \\
\frac{k^{n}-1}{k-1}<\frac{m-1}{k-1} \leqslant \frac{k^{n+1}-1}{k-1}, \\
k^{n}<m \leqslant k^{n+1} .
\end{gathered}
$$

Следовательно, $n+1=] \log _{k} m\left[\right.$. В силу леммы 9 (или 8) $\left|S_{2}\right|=2 m_{1}-2 n$. 
Подсхема $S_{3}$ состоит из единственного изолированного $m$-го входа. Ясно, что

$$
\begin{aligned}
|S| & =m_{1}(k-2)+m_{2}-1+2 m_{1}-2 n \\
& =m_{1}(k-1)+m_{1}+m_{2}+1-2 n-2 \\
& =m-1+](m-1) /(k-1)[-2] \log _{k} m[.
\end{aligned}
$$

Покажем, что построенная схема порождает матрицу без совпадающих столбцов. Рассмотрим два столбца порожденной матрицы, $i$-й и $j$-й. Будем полагать, что $j<i$ и $] i /(k-1)[=] j /(k-1)[$.

Пусть $\pi=\left(\begin{array}{llll}1 & 2 & \ldots & k-1\end{array}\right)$ - цикл из симметрической группы $S_{k-1}$. Из конструкции построенной схемы и леммы 5 легко следуют соотношения, связывающие значения $p_{t}$ и $q_{t}$ ненулевых $t$-х компонент $i$-го и $j$-го столбцов:

$$
p_{t}=\pi^{(i-j)} q_{t}
$$

Так как $0<i-j<k-1$, очевидно, что рассматриваемые столбцы различны.

Рассмотрим случай $] i /(k-1)[>] j /(k-1)[$. Пусть

$$
i=i_{1}(k-1)+i_{0}, \quad j=j_{1}(k-1)+j_{0}, \quad 0<i_{0}<j_{0}<k-1 .
$$

В силу соотношения (1) значения $p_{t}$ и $p_{t}^{\prime}$ ненулевых $t$-х компонент $i$-го и $\left(i-i_{0}+1\right)$-го столбцов связаны соотношением

$$
p_{t}=\pi^{\left(i_{0}-1\right)} p_{t}^{\prime}
$$

а значения $q_{t}$ и $q_{t}^{\prime}$ ненулевых $t$-х компонент $j$-го и $\left(j-j_{0}+1\right)$-го столбцов соотношением

$$
q_{t}=\pi^{\left(i_{0}-1\right)} q_{t}^{\prime}
$$

Следовательно, справедливо равенство

$$
p_{t}^{\prime}=\pi^{\left(j_{0}-i_{0}\right)} q_{t}^{\prime}
$$

Если разность $j_{0}-i_{0}$ отлична от нуля, то очевидно, что последние ненулевые компоненты $\left(j-j_{0}+1\right)$-го и $\left(i-i_{0}+1\right)$-го столбцов не могут быть одновременно равны единице. Но по построению эти столбцы входят в матрицу $C^{\prime}\left(n, m_{1}+1\right)$, то есть их последние ненулевые компоненты равны единице. Пришли к противоречию. Если $j_{0}-i_{0}=0$, то $\left(j-j_{0}+1\right)$-й и $\left(i-i_{0}+1\right)$-й столбцы совпадают. Но матрица $C^{\prime}\left(n, m_{1}+1\right)$ состоит из попарно различных столбцов. Вновь получаем противоречие. Таким образом, показано, что построенная схема порождает матрицу без совпадающих столбцов. Верхняя оценка теоремы доказана. Нижняя оценка следует из леммы 2. Теорема доказана полностью.

Обозначим через $s_{i}(x)$ (произвольную) функцию, удовлетворяющую условиям $s_{i}(0)=0, s_{i}(1)=i$.

Лемма 10. Пусть $W_{n} \in W\left(k, n, k^{n}\right)$. Тогда

$$
V_{B_{k, 2}}(B(k, n)) \leqslant 2 k^{n}-2 n-2 \text {. }
$$


Доказателъство. Построим схему $S_{k, n}$, порождающую матрицу $W_{n}$ со сложностью $2 k^{n}-2 n-2$. Без ограничения общности будем полагать, что $j$-й столбец матрицы $W_{n}$ совпадает с $k$-ичным разложением числа $j-1$. Строить схему $S_{k, n}$ при произвольных $k \geqslant 2$ и $n \geqslant 1$ будем индукцией по $n$. Основанием индукции будет схема $S_{k, 1}$. Эта схема порождает единственную строку матрицы $W_{1}$ в соответствии с формулой

$$
x_{2} \vee s_{2}\left(x_{3}\right) \vee \ldots \vee s_{k-1}\left(x_{k}\right) \text {. }
$$

Очевидно, что

$$
\left|S_{k, 1}\right|=2(k-1)-2 .
$$

Предположим далее, что при некотором $n \geqslant 1$ и при произвольном $k \geqslant 2$ существует схема $S_{k, n}$, порождающая матрицу $W_{n}$ из $W\left(k, n, k^{n}\right)$ с требуемой сложностью. На основе этой схемы построим схему $S_{k, n+1}$, порождающую матрицу $W_{n+1}$ из $W\left(k, n+1, k^{n+1}\right)$. Схема $S=S_{k, n+1}$ будет состоять из подсхем $S_{1}, S_{2}, S_{3}, S_{4}$. Опишем эти подсхемы.

Подсхема $S_{1}$ порождает все векторы

$$
y_{i}=\bigvee_{j} x_{j}, \quad(i-1) k^{n}<j \leqslant i k^{n}, \quad 2 \leqslant i \leqslant k .
$$

Подсхема $S_{2}$ порождает все векторы

$$
z_{i}=\bigvee x_{i+j k^{n}}, \quad 0 \leqslant j \leqslant k-1, \quad 2 \leqslant i \leqslant k^{n} .
$$

Подсхема $S_{3}$ является экземпляром схемы $S_{k, n}$. $\mathrm{K} i$-му входу $S_{3}$ подключается вектор $z_{i}$, порождаемый подсхемой $S_{2}$. Легко видеть, что подсхема $S_{3}$ порождает последние $n$ строк матрицы $W_{n+1}$.

Подсхема $S_{4}$ порождает первую строку матрицы $W_{n+1}$, исходя из векторов $y_{i}$, порожденных подсхемой $S_{1}$, в соответствии с формулой

$$
y_{2} \vee s_{2}\left(y_{3}\right) \vee \ldots \vee s_{k-1}\left(y_{k}\right) \text {. }
$$

Оценим сложность схемы $S$. Легко видеть что

$$
\begin{aligned}
|S| & =\sum_{i=1}^{4}\left|S_{i}\right|=\left(k^{n}-1\right)(k-1)+\left(k^{n}-1\right)(k-1)+\left|S_{k, n}\right|+(2 k-4) \\
& =\left|S_{k, n}\right|+2\left(k^{n+1}-k^{n}\right)-2 .
\end{aligned}
$$

По предположению индукции $\left|S_{k, n}\right|=2\left(k^{n}-1\right)-2 n$, поэтому

$$
\left|S_{k, n+1}\right|=2\left(k^{n}-1\right)-2 n+2 k^{n}(k-1)-2=2\left(k^{n+1}-1\right)-2(n+1) .
$$

Лемма доказана.

Теорема 2. При любом $m>1$ существует такая матрица $W \in W(k,] \log _{k} m[, m)$, чmo

$$
\left.V_{B_{k, 2}}(W)=2 m-2\right] \log _{k} m[-2 \text {. }
$$


Доказательство. Будем полагать, что $m$ не является целой степенью $k$, в противном случае существование требуемой матрицы следует из лемм 10 и 3 . Положим $n=\left[\log _{k} m\right]$. Пусть $W^{\prime} \in W\left(k, n, k^{n}\right)$ и пусть $S^{\prime}$ - построенная в доказательстве леммы 10 схема, порождающая $W^{\prime}$. Воспользуемся этой схемой для построения схемы $S$, порождающей матрицу $W$. Схема $S$ состоит из подсхем $S_{1}, S_{2}, S_{3}, S_{4}$. Опишем эти подсхемы.

Подсхема $S_{1}$ порождает все векторы

$$
\left.y_{i}=\bigvee x_{j}, \quad(i-1) k^{n}<j \leqslant \min \left(i k^{n}, m\right), \quad 2 \leqslant i \leqslant\right] m / k^{n}[.
$$

Подсхема $S_{2}$ порождает все векторы

$$
z_{i}=\bigvee x_{i+j k^{n}}, \quad 0 \leqslant j \leqslant k-1, i+j k^{n} \leqslant m, \quad 2 \leqslant i \leqslant k^{n} .
$$

Подсхема $S_{3}$ является экземпляром схемы $S^{\prime}$. К $i$-му входу $S_{3}$ подключается вектор $z_{i}$, порождаемый подсхемой $S_{2}$. Легко видеть, что подсхема $S_{3}$ порождает последние $n-1$ строк матрицы $W$.

Подсхема $S_{4}$ порождает первую строку матрицы $W$, исходя из векторов $y_{i}$, порожденных подсхемой $S_{1}$, в соответствии с формулой

$$
y_{2} \vee s_{2}\left(y_{3}\right) \vee \ldots \vee s_{k-1}\left(y_{k}\right) .
$$

Оценим сложность схемы $S$. Ясно, что

$$
\begin{aligned}
|S|= & \sum_{i=1}^{4}\left|S_{i}\right| \\
= & \left.m-k^{n}-\right]\left(m-k^{n}\right) / k^{n}\left[+(] m / k^{n}[-1)\left(m-(] m / k^{n}[-1) k^{n}-1\right)\right. \\
& +(] m / k^{n}[-2)\left(k^{n}-\left(m-(] m / k^{n}[-1) k^{n}\right)\right)+2 k^{n}-2 n-2+2(]\left(m-k^{n}\right) / k^{n}[-1) \\
= & 2 m-2(n+1)-2 .
\end{aligned}
$$

Таким образом, верхняя оценка, указанная в теореме, доказана. Нижняя оценка следует из леммы 3. Теорема доказана полностью.

Пусть $W(m) \in W(k, n, m)$ и $j$-й столбец $W(m)$ совпадает с $k$-ичным представлением числа $j-1$. Через $N W(m)$ обозначим матрицу из $W(k, n, m)$, у которой $j$-й столбец совпадает с $k$-ичным представлением числа $k-j$. Пусть $D(m)-$ матрица размера 2] $\log _{k} m[\times m$, у которой $i$-я строка при $1 \leqslant i \leqslant] \log _{k} m$ [ совпадает с $i$-й строкой матрицы $W(m)$, а при $] \log _{k} m[+1 \leqslant i \leqslant 2] \log _{k} m[$ с $i-] \log _{k} m[)$-й строкой матрицы $N W(m)$.

Теорема 3. При любом $m>1$ справедливо равенство

$$
V_{B_{k, 3}}(D(m))=2 m+O\left(m^{1 / 2}\right) .
$$

Доказательство. Введем четыре множества индексов $I_{1}, I_{2}, I_{3}, I_{4}$, полагая

$$
\begin{aligned}
& \left.I_{1}=\{1, \ldots,] 1 / 2\right] \log _{k} m[[\}, \\
& \left.I_{2}=\{] 1 / 2\right] \log _{k} m\left[[+1, \ldots,] \log _{k} m[\},\right. \\
& \left.I_{3}=\{] \log _{k} m[+1, \ldots,] \log _{k} m[+] 1 / 2\right] \log _{k} m[[\}, \\
& \left.I_{4}=\{] \log _{k} m[+] 1 / 2\right] \log _{k} m\left[[+1, \ldots, 2] \log _{k} m[\} .\right.
\end{aligned}
$$


Построим схему $S_{m}$, порождающую матрицу $D(m)$. Схема $S_{m}$ состоит из двух подсхем $S_{m, 1}$ и $S_{m, 2}$.

Подсхема $S_{m, 1}$ порождает матрицу $D_{1}$, состоящую из строк матрицы $D(m)$, номера которых принадлежат множествам $I_{1}$ и $I_{3}$. Подсхема $S_{m, 2}$ порождает матрицу $D_{2}$, состоящую из строк матрицы $D(m)$, номера которых принадлежат множествам $I_{2}$ и $I_{4}$. Легко видеть, что матрица $D_{1}$ содержит не более $k^{\left|I_{1}\right|}$, а матрица $D_{2}$ не более $k^{\left|I_{2}\right|}$ различных столбцов.

Конструкции подсхем $S_{m, 1}$ и $S_{m, 2}$ практически одинаковы, поэтому ограничимся описанием только подсхемы $S_{m, 1}$. Подсхема $S_{m, 1}$ состоит из двух подсхем $S_{m, 1}^{1}$ и $S_{m, 1}^{2}$.

Подсхема $S_{m, 1}^{1}$ порождает все векторы

$$
y_{i}=\bigvee x_{j}
$$

где

$$
\left.(i-1) k^{\left|I_{1}\right|} \leqslant j \leqslant \max \left(i k^{\left|I_{1}\right|}, m\right), \quad 1 \leqslant i \leqslant\right] m / k^{\left|I_{1}\right|}[.
$$

Подсхема $S_{m, 1}^{2}$ является экземпляром схемы $S$, порождающей матрицу

$$
B(k,] 1 / 2] \log _{k} m[[) \text {. }
$$

К $i$-му входу $S$ подключается вектор $y_{i}$, порождаемый подсхемой $S_{m, 1}^{1}$.

Оценим сложность подсхемы $S_{m, 1}$. Легко видеть, что

$$
\left|S_{m, 1}\right|=\left|S_{m, 1}^{1}\right|+\left|S_{m, 1}^{2}\right| \leqslant m+c k^{1 / 2] \log _{k} m[l}
$$

где $c$ - постоянная. Так как $\left|S_{m}\right|=\left|S_{m, 1}\right|+\left|S_{m, 1}\right|$, то

$$
\left|S_{m}\right| \leqslant 2 m+2 c k^{1 / 2] \log _{k} m[l} \text {. }
$$

Таким образом, верхняя оценка, указанная в теореме, доказана. Нижняя оценка следует из леммы 4. Теорема доказана полностью.

\section{4. Сложность матриц и сложность функций}

Сложность функции $k$-значной логики $f$ при вычислении ее схемами в базисе $B$ обозначим через $L_{B}(f)$.

Пусть $W$ - матрица размера $n \times m, w$ - частичная функция от $r=r_{1}+r_{2}$ переменных, где $\left.r_{1}=\right] \log _{k} n\left[, r_{2}=\right] \log _{k} m[$.

Будем говорить, что матрица $W$ соответствует функции $w$, а функция $w$ соответствует матрице $W$, если при всех $i, j$ таких, что $1 \leqslant i \leqslant n, 1 \leqslant j \leqslant m$, справедливо равенство

$$
W_{i, j}=w\left(\alpha_{1}, \ldots, \alpha_{r_{1}}, \beta_{1}, \ldots, \beta_{r_{2}}\right),
$$

где $\alpha$ и $\beta$ определяются из условий

$$
i=\sum_{s=1}^{r_{1}} k^{s-1} \alpha_{s}, \quad j=\sum_{s=1}^{r_{2}} k^{s-1} \beta_{s} .
$$


Через $J=\left\{J_{1}, \ldots, J_{r_{2}}\right\}$ обозначим систему векторов, $i$-й вектор которой совпадает с $i$-й строкой матрицы $J(m) \in W\left(k, r_{2}, m\right)$. Через $D J=\left\{J_{1}, \ldots, J_{2 r_{2}}\right\}$ обозначим систему векторов, $i$-й вектор которой совпадает с $i$-й строкой матрицы $D(m)$.

Нетрудно убедиться, что всякая $(B, J)$-схема $S$, порождающая матрицу $W$, будет также вычислять систему функций $w_{r_{2}}\left(u_{1}, \ldots, u_{r_{2}}\right)$ при условии, что $i$-я функция этой системы $w_{r_{2}, i}$ соответствует $i$-й строке $W$, а к каждому входу схемы $S$ вместо вектора $J_{j}$ подключена $j$-я переменная системы функций $w_{r_{2}}$. Аналогичное утверждение справедливо и для $(B, D J)$ - схемы.

Лемма 11. Если множество векторов $G_{2}$ может быть порождено $\left(B, G_{1}\right)$-схемой, mo

$$
V_{B, G_{1}}(W) \leqslant V_{B, G_{1}}\left(G_{2}\right)+V_{B, G_{2}}(W)
$$

Доказателъство. Пусть $S$ - схема, порождающая матрицу $W$, исходя из множества векторов $G_{2}=\left\{G_{21}, \ldots, G_{2 t}\right\}, S^{\prime}-$ схема, порождающая множество векторов $G_{2}$, исходя из множества векторов $G_{1}$. Подключим $j$-й выход схемы $S^{\prime}$ к $j$-му входу $S$. В результате получим схему, порождающую матрицу $W$, исходя из множества векторов $G_{1}$, сложность которой удовлетворяет утверждению леммы. Лемма доказана.

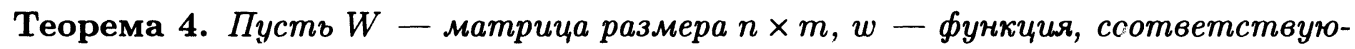
щая матрице $W$. Тогда

$$
L_{B_{k, 1}}(w) \geqslant \frac{1}{n}\left(V_{B_{k, 1}}(W)-(m-1+](m-1) /(k-1)[-2 n)\right) .
$$

Доказательство. Воспользуемся леммой 11 и теоремой 1. Легко видеть, что

$$
\begin{aligned}
L_{B_{k, 1}}(w) & \geqslant \max _{i} L_{B_{k, 1}}\left(w_{r_{2}, i}\right) \geqslant \frac{1}{n} L_{B_{k, 1}}\left(w_{r_{2}}\right)=\frac{1}{n} V_{B_{k, 1} J}(W) \\
& \geqslant \frac{1}{n}\left(V_{B_{k, 1}}(W)-(m-1+](m-1) /(k-1)[-2 n)\right) .
\end{aligned}
$$

Теорема доказана.

Аналогично доказывается следующая теорема. Для ее доказательства вместо теоремы 1 необходимо воспользоваться теоремой 2.

Теорема 5. Пусть $W$ - матрича размера $n \times m, w-$ функция, соответствующая матрище $W$. Тогда

$$
L_{B_{k, 2}}(w) \geqslant \frac{1}{n}\left(V_{B_{k, 2}}(W)-(2 m-2] \log _{k} m[-2)\right) .
$$

Далее докажем теорему 6, связывающую монотонную сложность порождения матриц и сложность реализации функций схемами в полном базисе. Пусть

$$
\begin{aligned}
& I_{j}(x)= \begin{cases}k-1, & \text { если } x=j, \\
0, & \text { если } x \neq j,\end{cases} \\
& N(x)=k-1-x .
\end{aligned}
$$


Известно, что система функций

$$
B=\left\{I_{1}(x), \ldots, I_{k-1}(x), 0, \ldots, k-1, \max (x, y), \min (x, y)\right\}
$$

является полной в $P_{k}$. Введем функцию $\bar{I}_{j}(x)$, полагая

$$
\bar{I}_{j}(x)= \begin{cases}k-1, & \text { если } x \geqslant j \\ 0, & \text { если } x<j .\end{cases}
$$

Так как

$$
I_{j}(x)=\min \left(\bar{I}_{j}(x), \bar{I}_{N(j)}(N(x))\right)
$$

система функций

$$
B_{2}=\left\{\bar{I}_{1}(x), \ldots, \bar{I}_{k-1}(x), 0, \ldots, k-1, N(x)=k-1-x, \max (x, y), \min (x, y)\right\}
$$

является полной в $P_{k}$.

Схему $S$ в базисе $B_{2}$ назовем приведенной, если в этой схеме символами $N$ отмечены только вершины, смежные входам $S$. Приведенной сложностью функции $f$ назовем величину $L_{2}^{\prime}(f)$, равную сложности минимальной приведенной схемы $S$, вычисляющей функцию $f$. Справедлива следующая лемма.

Лемма 12. Справедливо неравенство

$$
L_{2}^{\prime}(f) \leqslant 2 L_{B_{2}}(f)+n
$$

где $n$ - число существенных переменных функции $f$.

Доказательство. Неравенство леммы основано на следующем предложении. Пусть $S$-- схема в базисе $B_{2}$ и пусть $F(S)=\left\{f_{i}\right\}$ - система всех функций, вычисляемых элементами $S$. Тогда сложность приведенной схемы $S^{\prime}$, вычисляющей систему функций $F^{\prime}(S)=\left\{f_{i}, N f_{i}\right\}$, не превосходит величины $2|S|+n$, где $n$ - число существенных переменых системы $F(S)$. Это предложение докажем индукцией по сложности $S$. При $|S|=0$ предложение очевидно. Предположим, что оно верно и при $|S|=m$. Покажем, что предложение справедливо и при $S=m+1$. Пусть $s$ - элемент схемы $S$ с выходной степенью, равной нулю, и пусть $S_{1}$ - схема $S$ без элемента $s$. По предположению индукции

$$
\left|S_{1}^{\prime}\right| \leqslant 2 m+n
$$

Существование приведенной схемы, вычисляющей систему функций $F^{\prime}(S)$ с требуемой сложностью, следует из (2) и из легко проверяемых тождеств

$$
\begin{aligned}
N(\max (x, y)) & =\min (N(x), N(y)), \\
N(\min (x, y)) & =\max (N(x), N(y)), \\
N\left(\bar{I}_{j}(x)\right) & =\bar{I}_{N(j-1)}(N(x)) .
\end{aligned}
$$

Лемма доказана.

Воспользовавшись леммой 12 и теоремой 3 , нетрудно доказать следующую теорему. 


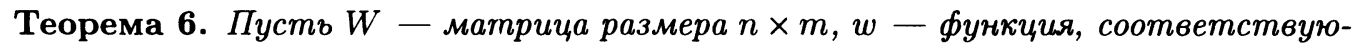
щая матриче $W$. Тогда

$$
L_{B_{2}}(w) \geqslant \frac{1}{2 n}\left(V_{B_{k, 3}}(W)-\left(2 m+O\left(m^{1 / 2}\right)\right)\right) .
$$

Теоремы 4-6 показывают, что доказательство сравнительно невысоких линейных нижних оценок сложности порождения узких матриц $k$-значной логики столь же трудно, как и доказательство нелинейных и даже экспоненциальных нижних оценок сложности вычисления функций $k$-значной логики схемами в полных базисах. Причем это справедливо и в случае порождения матриц монотонными схемами. Рассмотрим этот случай подробно. Пусть $W$ - матрица размера $n \times m$, где $n=\left[m /\left(g(m) \log _{k} m\right)\right], g(m)$ - некоторая неограниченно возрастающая функция, $w$ - функция, соответствующая матрице $W$. Предположим, что справедливо неравенство

$$
V_{B_{k, 3}}(W) \geqslant(2+\varepsilon) m .
$$

В этом случае из теоремы 6 следует, что

$$
L_{B_{2}}(w) \geqslant \frac{\varepsilon}{2} g(m) \log _{k} m\left(1+O\left(m^{-1 / 2}\right)\right)
$$

Так как функция $w$ зависит от $\left.r=r_{1}+r_{2} \leqslant 2\right] \log _{k} m[$ аргументов, для этой функции при $m$, большем некоторого $m_{0}$,

$$
L_{B_{2}}(w) \geqslant \operatorname{crh}(r),
$$

где $c$ - постоянная, а $h$ - неограниченно возрастающая функция. Легко видеть, что правая часть последнего неравенств нелинейна относительно $r$.

Если $n=\left[m^{1-\delta}\right]$ для некоторой постоянной $\delta, 0<\delta<1$, то нетрудно убедиться в том, что

$$
L_{B_{2}}(w) \geqslant \frac{\varepsilon}{2} m^{\delta}\left(1+O\left(m^{-1 / 2}\right)\right) .
$$

Как и в предыдущем случае, функция $w$ зависит от $r \leqslant 2] \log _{k} m[$ аргументов, поэтому при $m$, большем $m_{0}$, справедливо неравенство

$$
L_{B_{2}}(w) \geqslant c_{1} 2^{\delta r / 2}
$$

где $c_{1}$ - постоянная. Очевидно, что правая часть неравенства экспоненциальна относительно $r$.

Автор благодарен О. Б. Лупанову за внимание к работе и Н. А. Карповой за ряд полезных замечаний.

\section{Список литературы}

1. Чашкин А. В. О сложности булевых матриц, графов и соответствующих им булевых функций. Дискреттая: математика (1994) 6, №2, 43-73.

2. Яблонский С. В. Введение в дискретную математику. Наука, Москва, 1979.

Статья поступила 21.03.1996. 